\title{
La fabrique pragmatique des politiques publiques
}

\section{Philippe Zittoun}

\section{(2) OpenEdition}

\section{Journals}

Electronic version

URL: http://journals.openedition.org/anthropodev/543

DOI: 10.4000/anthropodev.543

ISSN: 2553-1719

Publisher

APAD - Association pour l'anthropologie du changement social et du développement

\section{Printed version}

Date of publication: 1 May 2017

Number of pages: 65-89

ISBN: 979-10-93476-04-9

ISSN: 2276-2019

\section{Electronic reference}

Philippe Zittoun, «La fabrique pragmatique des politiques publiques », Anthropologie \& développement [Online], 45 | 2017, Online since 01 November 2017, connection on 01 May 2019. URL : http:// journals.openedition.org/anthropodev/543 ; DOI : 10.4000/anthropodev.543

\section{(c) (1)}

La revue Anthropologie \& développement est mise à disposition selon les termes de la Licence Creative Commons Attribution 4.0 International. 


\title{
La fabrique pragmatique des politiques publiques
}

\author{
Philippe Zittoun
}

Cet article expose les principaux principes, concepts et hypothèses d'une approche pragmatique des politiques publiques. Inspirée par la philosophie pragmatique américaine de Peirce, James et Dewey du début du $20^{e}$ siècle, par le linguistic turn de la philosophie des années 1970 et par la sociologie pragmatique française de Boltanski et Latour, cette nouvelle perspective privilégie la description concrète des activités que conduisent les acteurs pour maintenir ou transformer les politiques publiques. Elle privilégie un regard micro décrivant précieusement les phénomènes empiriques dans leur complexité à un regard macro rendu possible par des théories causales dont la force explicative a tendance à trop fortement simplifier la réalité. Prenant au sérieux les capacités cognitives et discursives des individus, elle propose de suivre la carrière des propositions d'action publique en s'intéressant aux stratégies définitionnelles, argumentatives et conflictuelles que ces derniers conduisent dans les jeux de langage, d'enrôlement et de pouvoir dans lesquels ils sont engagés. S'appuyant sur une méthode d'enquête qualitative, cette approche compréhensive accorde une importance majeure au sens qu'élaborent les acteurs pour définir les contraintes et les opportunités dans leurs interactions au cours du processus décisionnel.

This article highlights the main principles, concepts and hypothesis for a pragmatic approach on public policy. Inspired by the pragmatic philosophy of Peirce, James and Dewey in the begining of the 20's, by the linguistic turn in philosophy in the 1970's and by the French pragmatic sociology of Boltanski and Latour, this new perspective emphasizes the concrete description of policymakers' activities. It allows to focus precisely on the empirical phenomena and not put a large part of these activities in darkness by using some casual theories generally macro. Taking seriously the discursive and cognitive abilities of actors, it suggests to follow the career of policy proposals by focusing on the definitional, argumentative and conflictual strategies that they lead in games of language, of 
enrollment and of power in which they are engaged. Based on qualitative method, this comprehensive approach attaches great importance on actors configuring the constraints and opportunities during their interactions in the decision making process.

\section{Introduction}

Nés après la Seconde Guerre mondiale aux États-Unis, les travaux académiques sur les politiques publiques n'ont cessé de se multiplier et de se propager dans le monde entier. Partant de références et de travaux initiatiques souvent communs, comme ceux d'Harold Lasswell (1951), de Charles Lindblom (1959) ou d'Aaron Wildavsky (1964), ces travaux se sont inscrits dans des approches et des paradigmes de plus en plus en fragmentés et éclatés. Il existe ainsi une multitude d'approches différentes dans le champ des politiques publiques. À cette diversité d'approches s'ajoute la spécificité des débats sectoriels et nationaux qui marquent les travaux de leurs empreintes. Les recherches sur les politiques publiques ne recouvrent ainsi pas les mêmes considérations en France, en Allemagne ou aux États-Unis, et suivant que l'on évoque les politiques de santé ou les politiques du logement, au point qu'il est de plus en plus difficile d'établir une problématique et un socle communs à ce champ.

Cette difficulté se retrouve dans la définition même de l'objet central, les politiques publiques. Lorsque Thomas Dye (1972) effectue un premier recensement dans les années 1970, il identifie déjà plus de quarante définitions différentes du concept et finit par conclure que le seul dénominateur commun entre toutes ces définitions n'est pas lié à son contenu spécifique mais à son producteur: il définit ainsi les politiques publiques comme ce que le gouvernement décide de faire ou de ne pas faire. Quarante ans plus tard, le nombre de définitions n'a cessé de croître mais le socle commun ne s'est pas pour autant élargi.

La problématique de recherche ne s'appuie pas non plus sur un socle commun. On peut identifier deux types de problématiques très différentes (Zittoun et Peters, 2016). Certains travaux privilégient la recherche normative de "policy analysis", c'est-à-dire de méthodes analytiques pour résoudre des problèmes publics. Les auteurs s'attachent à la production de connaissances normatives et atemporelles, d'instruments 
cognitifs et de méthodes analytiques qui ont vocation à agir sur le processus et qui sont généralement destinés aux policymakers (Bardach et Patashnik, 2015). D'autres travaux privilégient une problématique plus empirique et compréhensive avec une entrée davantage tournée vers les processus. On parle notamment des "policy process studies" dont la vocation première est de comprendre les processus de fabrique ou de mise en œuvre (Hudson et Lowe, 2014 ; Kingdon, 2010 ; Jones, 1984).

Inspirées par les travaux initiatiques américains, les approches françaises se sont développées à partir de la fin des années 1970 pour former une sous-discipline de la science politique. Bien que les premiers chercheurs français aient utilisé le terme "analyse des politiques publiques " pour désigner ce champ, on peut dire que la tradition française s'inscrit dès le début dans les "policy process studies "; c'est-à-dire une approche qui privilégie la compréhension des phénomènes de fabrique et de mise en œuvre avec un regard largement inspiré de la sociologie des organisations et un intérêt marqué pour étudier le rôle des idées et des représentations (Zittoun et Demongeot, 2010) ${ }^{1}$. Bien qu'alimentées régulièrement par les travaux internationaux importés en France, les approches françaises ont développé leur propre débat et faiblement contribué aux débats internationaux jusqu'à récemment. Le choix de privilégier un concept comme celui d' " action publique " (Lascoumes et Le Galès, 2007) plutôt que celui de "politique publique " par exemple, considérant que ce dernier était trop associé à l'État centralisateur et à une cohérence qui peut biaiser le regard du chercheur, est un débat uniquement franco-français; les anglo-saxons n'ayant jamais considéré que le terme "public policy " était associé à un État centralisé (Halpern, Hassenteufel et Zittoun, à paraître).

Différents courants se distinguent au sein de cette "French touch" dans l'analyse des politiques publiques (Boussaguet et al., 2015). L'approche pragmatique développée dans cet article propose de

${ }^{1}$ En raison d'une mauvaise traduction au moment de l'importation, ce terme continue d'apporter de la confusion. On gardera donc le terme anglais pour éviter toute confusion avec son usage français ou on utilisera celui de "policy design ». Sur le sujet, voir notamment Halpern, Hassenteufel et Zittoun (à paraître). 
s'interroger sur le parcours des propositions de politiques publiques et leur mise à l'ordre du jour dans les processus décisionnels. Si la plupart des approches du processus de politiques publiques s'accordent à considérer la mise à l'ordre du jour médiatique d'un problème public comme la résultante d'un travail spécifique de définition et de propagation (Neveu, 2015), elles n'accordent pas la même attention à la définition et à la propagation des solutions. L'approche pragmatique propose ainsi de saisir le processus de fabrique des politiques publiques, d'abord comme le résultat d'une activité politique des acteurs en interaction dans les arènes politico-administratives. Cherchant tout à la fois à définir, à propager et à imposer une solution, ces acteurs chaînent ensemble un instrument d'action avec un problème insoluble, une société désordonnée, un " décideur » capable de remettre de l'ordre et une coalition en soutien.

Définir les politiques publiques pour donner du sens à leur changement

Dès les premiers travaux sur les politiques publiques, les chercheurs ont essayé de définir l'objet "politique publique ». II s'agissait pour eux de mieux cerner l'objet et d'en définir les contours pour ensuite mieux l'étudier. Si l'on se réfère au travail inaugural d'Harold Lasswell dans les années 1950, ce dernier définit une politique publique comme un " choix important" mais libéré de toute connotation partisane ${ }^{2}$. Le concept anglais de " policy " se veut alors une façon plus neutre et plus apolitique d'aborder les questions de politiques publiques. Mais là où l'anglais utilise plusieurs termes pour distinguer les activités partisanes des activités de politique publique, le français, comme de nombreuses langues, utilise un seul terme, celui de "politique ". Toutefois, cette définition - comme toutes celles produites ensuite - suscite une large insatisfaction et conduit les chercheurs à une multiplication des définitions possibles. Chaque ouvrage et chaque manuel disposent ainsi de leur propre définition du

${ }^{2}$ "The word "policy" is commonly used to designate the most important choices [...]. [It] is free of many of the undesirable connotations clustered about the world political which is often believed to imply "partisanship" or "corruption" " (Laswell, $1951: 2$ ). 
concept de politique publique. Pour autant, à défaut d'une définition commune, il reste possible d'identifier une série de caractéristiques que partagent les chercheurs. Sans prétendre à une quelconque exhaustivité, on retiendra principalement de ces définitions cinq caractéristiques majeures.

Tout d'abord, en reprenant la définition de Thomas Dye (1972: 3), "Whatever governments choose to do or not do", les chercheurs considèrent qu'une politique publique est d'abord un concept empirique destiné à décrire l'action concrète de l'État (ou d'une autorité publique) ou autrement dit l'État en action (Jobert et Muller, 1987). Plus exactement, si le concept générique "politique publique " peut être considéré comme théorique, sa déclinaison thématique (i.e. la politique du logement, la politique économique, etc.) et territoriale (nationale, régionale, etc.) correspond à une réalité empirique que les chercheurs tout autant que les acteurs utilisent.

La deuxième caractéristique est de considérer qu'une politique publique n'est jamais composée d'un seul élément, mais forme un groupement d'éléments divers tels que des décisions, des lois, des décrets, des circulaires, des budgets, des institutions, des fonctionnaires, des activités, des discours, etc. Hugh Heclo (1972) évoque ainsi le fait qu'une politique publique est toujours bien plus qu'une simple décision, elle est un ensemble de taille intermédiaire ${ }^{3}$. Guy Peters (2015) considère qu'une politique publique est un regroupement d'activités engagées par l'État ${ }^{4}$. C'est là que le concept se complexifie car, s'il est possible de repérer empiriquement chaque élément pris isolément, le regroupement d'éléments épars pose problème dans la mesure où il n'est jamais observable empiriquement en tant qu'ensemble. Une politique publique reste donc une construction qu'acteurs comme chercheurs peuvent

\footnotetext{
${ }^{3}$ "The term policy is usually considered to apply to something "bigger" than particular decisions, but "smaller" than general social movements. Thus, policy, in term of level of analysis, is a concept placed roughly in the middle rang " (Heclo, $1972: 84$ ).

${ }^{4}$ " Public Policy is the set of activities that governments engage in for the purpose of changing their economy and society. [...] The study of public policy is understanding what governments do and their effects on citizens" (Peters, 2015 : 12).
} 
effectuer de nombreuses façons différentes. Une même action, comme par exemple la mise en place de la réduction de la vitesse automobile, peut être considérée tout autant comme un élément de la politique des déplacements ou de la politique environnementale.

La troisième caractéristique renvoie à la question du sens et de I'intentionnalité de cet ensemble. Pour de nombreux auteurs, une politique publique renvoie à une intentionnalité ou à un objectif. Hugh Heclo tout comme Guy Peters insistent ainsi sur la dimension intentionnelle d'une politique publique ${ }^{5}$. Richard et Smith évoquent

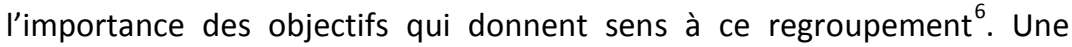
politique publique est donc d'abord un regroupement qui fait sens et qui s'organise autour d'orientations. La question qui reste controversée est celle de savoir qui élabore ce sens et si ce sens est objectivable. Pour les uns, cela reste le chercheur qui doit effectuer le regroupement et établir la cohérence alors que, pour les autres, il s'agit d'observer le travail définitionnel des acteurs qui donnent du sens. Paradoxalement, de nombreux chercheurs français, qui s'inscrivent pourtant dans une tradition constructiviste et qui accordent de l'importance au sens de l'action élaboré par les acteurs, ont voulu se débarrasser du concept de politique publique au profit de celui d'action publique en suggérant que le premier était trop chargé de sens et qu'il finissait par biaiser l'observation (Commaille, 2004). En faisant cela, ils négligent le travail de mise en sens que produisent les acteurs en utilisant fréquemment le concept de politique publique.

La quatrième caractéristique est de considérer qu'une politique publique ne peut se comprendre sans prendre en compte le processus dans lequel elle prend place et au cours duquel de nombreux acteurs sont impliqués (Jones, 1984 ; Anderson, 1979). Les chercheurs ont identifié plusieurs phases plus ou moins autonomes dont nous ne citerons ici que les quatre principales (Kingdon, 2010): la mise à l'ordre du jour du

${ }^{5}$ " A second and essential element in most writers" use of the term is purposiveness of some kind » (Heclo, 1972).

${ }^{6}$ "Policy is a general term used to describe a formal decision or plan of action adopted by an actor [...] to achieve a particular goal » (Richards et Smith, 2002). 
problème ${ }^{7}$, la formulation de la proposition, le processus décisionnel et la mise en œuvre. Au cours de chacune des phases, la coopération des acteurs constitue un des problèmes majeurs auxquels ces derniers doivent faire face. En pratique, ces phases peuvent se chevaucher, certaines peuvent manquer.

La cinquième caractéristique est la mise en évidence d'effets propres et inattendus pour chacune de ces phases (Stone, 2001). Quelles que soient les intentions des acteurs et leur position institutionnelle, l'émergence d'un problème, la dynamique d'une solution et la mise en œuvre leur échappent toujours en partie. Si la mise en œuvre d'une solution est sans aucun doute le phénomène qui génère le plus de conséquences imprévisibles, les processus de mise à l'ordre du jour des problèmes et d'émergence des solutions répondent également à des dynamiques incertaines. C'est pourquoi il est essentiel de distinguer les intentions qu'un acteur place dans une proposition à un temps t et les effets que la solution génère une fois mise en place. Cette incertitude des effets s'accompagne d'une incapacité des acteurs à s'accorder sur la mesure de ceux-ci, laissant place à une multitude d'interprétations concurrentes.

$\mathrm{Si}$ ces cinq caractéristiques sont relativement partagées par les chercheurs, il n'en reste pas moins qu'elles ne suffisent pas à créer un consensus autour de la définition d'une politique publique. À chaque tentative d'objectivation d'une définition, émergent des critiques et des définitions concurrentes. Pourtant, les chercheurs, confrontés à une impossibilité relativement similaire concernant la définition objective des problèmes, ont préféré abandonner ce travail définitionnel pour mieux observer celui que conduisent les acteurs engagés dans le processus (Spector et Kitsuse, 1973). Ce déplacement de l'attention sur les définitions subjectives et concurrentes des problèmes publics a permis de sortir de l'impasse définitionnelle dans laquelle se trouvaient les chercheurs, mais ils n'ont pas fait de même pour les solutions.

\footnotetext{
${ }^{7}$ Si les anglophones utilisent le terme " agenda ", sa traduction correcte en français n'est pas " agenda » mais « ordre du jour ».
} 
Nous voudrions considérer une politique publique comme un assemblage d'éléments hétérogènes, sans liens évidents ou logiques, auxquels les acteurs tentent de donner du sens et de la cohérence en fonction d'intentions et de stratégies contingentes. D'une certaine façon, on peut emprunter à Michel Foucault le concept de "dispositif " qu'il utilise pour parler de la prison, montrant que l'emprisonnement relie ensemble des éléments aussi hétérogènes que des mesures, des lois, des discours sur la criminalité, des "gardiens" et des "criminels", des institutions, etc. (Foucault, 1975). Il est possible d'importer cette définition tant une politique publique, comme la politique pénale, peut être d'abord considérée comme un assemblage d'éléments aussi hétérogènes que des lois, des décrets, des rôles sociaux, des organisations, des fonctionnaires, des subventions. Le rôle du chercheur n'est alors plus de définir une politique publique mais de s'intéresser à la façon dont les acteurs regroupent ces éléments hétérogènes et leur donnent du sens.

II revient dès lors aux chercheurs d'observer comment et à quelle occasion les acteurs définissent une politique publique. Un des moments les plus importants que nous avons pu observer au cours de nos propres travaux empiriques, c'est que cela se produit justement au moment où des acteurs veulent imposer une nouvelle solution qui vient réformer ou consolider une politique publique en place. Autrement dit, c'est d'abord autour d'un travail de légitimation d'un changement que s'effectue ce travail définitionnel (Zittoun, 2013).

Une approche pragmatique de l'action publique pour enquêter sur la fabrique des solutions

On l'a dit, on peut distinguer deux grandes traditions de recherche sur les politiques publiques. La " policy analysis " anglo-saxone est considérée soit comme une science appliquée élaborée pour résoudre les maux de la société (Lasswell, 1971), soit comme un art ou un savoir-faire (Wildavsky, 1987). Il s'agit de développer des méthodes qui permettent de résoudre des problèmes publics particulièrement complexes. Les auteurs qui s'inscrivent dans cette tradition parlent d'ailleurs de plus en plus de " policy design " pour intégrer cette dimension artistique à l'élaboration d'instruments et souligner que la dimension technique ne peut suffire 
(Peters, 2015 ; Howlett, 2009). Parmi les méthodes analytiques les plus célèbres, on peut évoquer la méthode rationnelle de type coût/avantage qui offre une grille méthodologique de résolution des problèmes complexes (Peters, 2015), ou encore celle de l'incrémentalisme disjoint (Lindblom, 1959) qui propose de construire une démarche de type essai/erreur pour résoudre les problèmes qui semblent insolubles.

Pour autant, cette dimension normative s'accompagne toujours d'une dimension compréhensive importante. Dans son dernier ouvrage sur le policy design par exemple, le premier conseil que donne Peters aux fabricants de politiques publiques, les policymakers, c'est de comprendre la complexité des politiques publiques que nous enseigne l'observation empirique des processus existants, et de saisir ainsi la pluralité des définitions d'un problème, l'absence de liens rationnels entre problème et solution, les nombreux effets inattendus et contradictoires d'instruments généralement ambigus et leur dimension politique irréductible (Peters, 2015).

À l'inverse, la tradition compréhensive se propose de comprendre sans juger la façon dont les politiques publiques se fabriquent, se transforment et se mettent en œuvre. Ce tournant s'opère dans les années 1970 avec la parution d'ouvrages centrés sur les processus de production des politiques publiques (Jones, 1984 ; Anderson, 1979 ; Lindblom et Woodhouse, 1992). II s'agit alors d'identifier des variables causales qui rendent compte de dynamiques propres aux politiques publiques. C'est ainsi que des théories telles que I'Advocacy Coalition Framework (ACF), qui propose de saisir le changement comme le résultat de conflits entre coalitions concurrentes, ou l'équilibre ponctué, qui suggère d'appréhender le changement à travers les perturbations que génère l'émergence de problèmes à l'agenda sur des politiques publiques relativement stables, proposent une compréhension considérée comme "objective" du changement (Sabatier, 1998). Pour autant, il est rare que ces travaux ne s'appuient pas sur des présupposés normatifs derrière leur revendication d'objectivité (Fischer et Gottweis, 2012). On trouve le plus souvent, d'une part, des appréciations très normatives sur les politiques publiques, définissant par exemple des normes de la réussite ou de l'échec, ce qu'est un changement et ce qu'il n'est pas, ou du moins sur son importance plus ou moins grande (Zittoun, 2009), mais aussi une véritable difficulté à prendre en compte les 
connaissances et les analyses subjectives que produisent et véhiculent les acteurs.

Paradoxalement, si les approches normatives s'appuient sur les approches compréhensives pour élaborer des stratégies d'analyse et de persuasion, peu de travaux compréhensifs prennent en considération le rôle des stratégies d'analyse, de mise en sens et de persuasion dans le processus. L'approche pragmatique propose de s'extraire de cet apparent paradoxe en décrivant empiriquement les opérations concrètes que conduisent les acteurs pour définir des problèmes et des solutions, pour mobiliser des catégories, des concepts, des contraintes. Plutôt que de considérer l'existence macro de forces ou de contraintes qui s'imposent aux acteurs, il s'agit de prendre au sérieux les opérations cognitives et discursives micro qu'ils conduisent pour effectuer une analyse qui leur permet d'associer une solution et un problème et de rendre visible des contraintes.

Herbert Simon est un des premiers à s'être intéressé à ces opérations concrètes que conduisent les acteurs pour résoudre les problèmes (Simon, 1997). Après avoir prouvé que les problèmes publics sont des problèmes bien trop complexes pour nos faibles capacités cognitives humaines et qu'ils ne peuvent faire l'objet d'une résolution analytique théorique et rationnelle, il en est venu à la conclusion que, pour résoudre malgré tout les problèmes, les individus doivent s'adapter à cette situation d'imperfection des connaissances. Simon compare la multiplicité des chemins possibles qui s'ouvrent au raisonnement, à un jeu d'échecs devant lequel le joueur, aussi fort soit-il, ne peut calculer en avance tous les coups pour déterminer son choix, et doit donc, en plus de ses capacités de calcul, mobiliser son intuition, utiliser des opérations de simplification et d'approximation, et des modes de raisonnement qui lui sont propres et qui le conduisent à une solution " satisfaisante».

Prolongeant les réflexions de Simon, Lindblom développe l'idée que, face à ces problèmes trop complexes pour être résolus rationnellement, les acteurs doivent "se débrouiller" et n'hésitent pas pour cela à développer des "stratagèmes" qui leur permettent de dépasser ces difficultés (Lindblom, 1959 ; Lindblom, 1979; Lindblom et Woodhouse, 1992). Il propose que les acteurs arrêtent de prétendre à une rationalité 
inatteignable et reconnaissent cette situation de débrouillardise comme un fait, pour mieux s'évertuer à un raisonnement rigoureux. Cette " science " de la débrouillardise le conduit à s'inspirer des travaux de Popper sur la démarche essai/erreur afin de construire le stratagème de l'incrémentalisme, du changement par petites touches, un modèle qui selon lui restera incompris (Dahl et Lindblom, 1963). Dans le prolongement des travaux de Lindblom, Wildavsky va aborder cette question de la pratique des policy analysis comme mode de résolution des problèmes en considérant lui aussi qu'il ne peut s'agir d'une science au sens traditionnel du terme mais plutôt d'un art (Wildavsky, 1987 ; Wildavsky, 2006).

Si ces auteurs sont convaincus du caractère illusoire du modèle rationnel dans les policy analysis, ils ne comprennent pas la fascination et l'attractivité d'un tel modèle. Lindblom a développé une critique en règle de ce qu'il nomme l'approche rationnelle mais se trouve démuni devant son succès. Wildavsky (1969), qui voit se développer les théories du choix rationnel et les méthodes de rationalisation des choix budgétaires, opposera à ces méthodes analytiques une analyse qualitative des politiques publiques qui tient compte de la complexité des problèmes. Ces modèles analytiques seront également mis à mal par la mise en évidence d'un autre phénomène au début des années 1970, autour du modèle dit " de la poubelle", le "garbage can model ". Ce modèle montre à quel point les décideurs, loin de mobiliser des stratagèmes de simplification pour résoudre de façon rationnelle les problèmes complexes, disposent le plus souvent de la solution avant même que n'émerge le problème (Cohen, March et Olsen, 1972). Autrement dit, les acteurs ont tendance à recycler des solutions anciennes pour traiter des problèmes nouveaux.

Mais ce modèle de la poubelle développé ensuite par John Kingdon (2010) dans le champ des politiques publiques conduit à une impasse sur la question du sens. Soit en effet l'association entre un problème et une solution n'est qu'une affaire de couplage et d'opportunité, mais dans ce cas on peut se demander pourquoi ces couplages ne conduisent jamais à des couples totalement absurdes, voire même pourquoi les solutions ont besoin de problème. Soit au contraire le couplage doit avoir un sens, mais dans ce cas, comment expliquer que les solutions existent avant les problèmes? 
Pour sortir de cette impasse, il faut revenir à une observation empirique de la façon dont les acteurs produisent ces couplages, réussissent mais aussi échouent à les chaîner l'un à l'autre. II faut s'interroger non seulement sur la fabrique du sens mais surtout sur le rôle que joue ce sens dans la fabrique des politiques publiques. Pour cela, on peut s'appuyer sur une approche pragmatique, héritage à la fois de la philosophie pragmatique américaine du début du $20^{\mathrm{e}}$ siècle (James, 1995 ; Dewey, 2010) et de la sociologie pragmatique française développée ces vingt dernières années (Boltanski, 2009) qui suggère de prendre au sérieux les compétences cognitives et définitionnelles des acteurs, et l'importance de ce qui se joue dans leurs interactions, comprises comme des espaces d'échanges au résultat incertain mais au cours desquelles les acteurs mettent à l'épreuve leur définition pour tenter de construire un accord autour d'un sens commun.

L'approche pragmatique accorde une importance essentielle à l'enquête et aux épreuves, c'est-à-dire à la façon dont les acteurs construisent leurs concepts, leurs définitions, leurs analyses, non pas seuls et isolés du monde, mais au cours d'interactions répétées avec d'autres. $\mathrm{Au}$ cours de ces interactions, ils mènent des "enquêtes" qui leur permettent d'identifier et de comprendre partiellement les concepts, les définitions et les analyses des autres, ils explicitent aussi en permanence leurs propres catégories, ils leur font subir des épreuves, à commencer par l'épreuve des critiques. Ces confrontations les amènent à éprouver leurs définitions, à les renforcer parfois, mais aussi à les adapter :

"La sociologie pragmatique a entrepris de décrire le monde social comme la scène d'un procès au cours duquel des acteurs en situation d'incertitude, procèdent à des enquêtes, consignent leur interprétation de ce qui se passe dans des rapports, établissent des qualifications et se soumettent à des épreuves" (Boltanski, 2009 : 48).

Analyser, c'est apprivoiser des problèmes "sauvages » pour les rendre traitables

Partant de ce constat, il nous faut nous intéresser plus en détail à la façon dont les acteurs procèdent pour définir les solutions qu'ils proposent, leur 
associer des problèmes et développer des analyses rationalisantes. L'approche pragmatique suggère de ne pas se contenter d'explication structurelle et macro pour rendre compte de ce processus de croisement entre un problème et une solution. L'observation des pratiques concrètes permet de montrer non seulement l'ingéniosité stratégique des acteurs pour coupler des problèmes et des solutions, mais aussi la façon dont ces couplages participent d'une redéfinition du problème et de la solution.

Pour bien comprendre ce processus, il nous faut partir d'exemples sur la façon dont les acteurs emboîtent les différents composants de leur énoncé. En France, il existe un débat en ce moment sur la proposition du gouvernement d'alléger les conditions de licenciement économique pour lutter contre le chômage important que connaît le pays. Pour justifier d'une telle position qui peut sembler contre-intuitive (licencier plus facilement pour faciliter l'embauche), les acteurs développent un raisonnement qui consiste à établir d'abord une sorte d'algorithme causal entre un problème, le chômage, et sa cause, les conditions de licenciement (généralement sous la forme : «si..., c'est que ») :

- $\quad$ si les patrons n'embauchent pas, c'est qu'ils ont peur de ne pas pouvoir licencier en cas de difficulté économique ;

- $\quad$ si les patrons ont peur de ne pas pouvoir licencier, c'est que le Code du travail est trop rigide ;

- or, réformer le droit du travail permet d'alléger les conditions de licenciement ;

- réformer le droit du travail permet donc de résoudre le problème du chômage.

Le problème (le chômage) est associé à une cause (la rigidité du règlement). De l'autre, la solution (réformer le règlement) est associée à une conséquence (alléger la règlementation). Ce double travail permet alors de créer un emboîtement apparemment simple et logique entre la cause du problème et la conséquence de la solution, ce qui par association indirecte permet d'établir un lien entre un problème et une solution. Toute I'habileté des acteurs repose sur ce que Wittgenstein (2014) nomme des jeux de langage, c'est-à-dire des jeux d'associations causales et normatives 
entre des propositions au départ distinctes, selon des règles souvent implicites, pour créer du sens.

Si comme nous l'avons évoqué précédemment, Wildavsky considère que la policy analysis est plus un art qu'une science, il ajoute que c'est moins l'art de résoudre des problèmes que celui de créer des problèmes qui peuvent être résolus (Wildavsky, 1987). L'approche pragmatique permet de compléter cette intuition en comparant la policy analysis d'abord à un jeu de langage au cours duquel les acteurs déploient des stratagèmes pour transformer un problème insoluble en problème traitable. Comme le souligne l'exemple, le stratagème essentiel consiste à opérer une double substitution : le problème par sa cause, une cause n'est rien d'autre qu'un problème traitable; la solution par sa conséquence. Dans l'exemple ci-dessus, la rigidité des règles de licenciement $n^{\prime}$ est pas seulement une "cause ", elle est surtout un nouveau problème qu'il faut régler mais un problème traitable cette fois-ci. D'une certaine façon, en associant une cause au problème, les acteurs ont réussi à dompter un problème "sauvage", c'est-à-dire un problème insoluble ${ }^{8}$, en le transformant en problème apprivoisé.

Pour conclure, et sans rentrer cette fois-ci dans tous les détails, la policy analysis doit plutôt être comprise comme un processus de production d'un énoncé de solution, c'est-à-dire d'un discours en recherche de stabilisation qui relie une solution à un problème à travers différents stratagèmes discursifs pour redéfinir le problème en le rendant traitable et en s'appuyant sur des arguments en charge de justifier une telle association. Il s'agit d'une connaissance pratique (les acteurs effectuent des assemblages en faisant appel à leur intuition ou à leur approximation), mais qui s'appuie aussi sur un travail de rationalisation et d'argumentation.

${ }^{8}$ Dans la littérature anglo-saxone, on parle souvent de « wicked problem » (Head, 2008). 
Les énoncés de politique publique face aux épreuves de l'argumentation et de la critique

L'approche pragmatique est souvent désignée comme une sociologie des épreuves, c'est-à-dire une sociologie qui s'attache à observer les pratiques des acteurs confrontés à des épreuves qu'ils doivent franchir (Barthe et al., 2014). Dès lors, elle rejette tout autant les approches sociologiques qui observent les jeux d'acteurs sans s'intéresser à ce qu'ils disent, que les approches d'analyse de discours qui décortiquent les discours sans prendre en compte les jeux d'acteurs dans lesquels ils prennent place. II s'agit ainsi de s'intéresser aux situations sociales concrètes au cours desquelles les acteurs produisent des énoncés de solutions pour comprendre dans quel contexte ceux-ci voient le jour, avec quelle intention, mais aussi d'en suivre le fil des évolutions.

Pour prendre l'exemple d'une recherche que j'ai conduite sur le tramway parisien (Zittoun, 2013), les premiers promoteurs du tramway se comptent au départ sur les doigts d'une main. Ils sont chacun au sein d'une organisation et appartiennent à des services d'étude dont la mission est de produire des propositions. Lorsque ces quelques promoteurs produisent des analyses pour justifier le tramway comme solution à des problèmes, ils le font d'abord parce qu'ils sont soumis à un impératif de persuasion et de justification. Ne pouvant prendre aucune décision seuls, ils doivent en effet d'abord persuader d'autres acteurs - à commencer par leurs supérieurs hiérarchiques - de l'intérêt d'un tel projet, dans un espace concurrentiel où des acteurs promouvant d'autres solutions sont eux-mêmes engagés dans des stratégies d'argumentation et de persuasion ${ }^{9}$.

Dans le cas du tramway, c'est d'abord l'incapacité des promoteurs à persuader d'autres acteurs qu'eux-mêmes qui permet de comprendre que les projets de tramways successifs finissent dans un rapport sur une étagère de bibliothèque poussiéreuse. Bruno Latour (1992) avait montré

\footnotetext{
${ }^{9}$ La sociologie des organisations a permis de souligner à quel point les organisations sont des espaces conflictuels où l'affrontement entre départements, entre ministères, entre bureaux est permanente et où la coopération des acteurs ne va pas de soi mais constitue un problème difficile à résoudre (Crozier et Friedberg, 1977).
} 
de la même façon qu'un projet a beau être absolument innovant, merveilleux technologiquement et prometteur, c'est d'abord la capacité de ses promoteurs à le propager en enrôlant des alliés qui lui permet de voir le jour. C'est ici que le problème vient jouer un rôle majeur. La principale différence entre le projet de tramway solution d'un problème de circularité et celui du tramway solution du problème de la pollution, c'est que le deuxième problème permet d'intéresser et d'enrôler un nombre bien plus grand d'acteurs à commencer par le maire de Paris, qui est interpelé sur cette question et doit trouver une façon d'y répondre. Le couplage n'est donc pas seulement un moyen pour les acteurs de réaliser un jeu de langage mais aussi d'intégrer un jeu d'acteurs, entre accords à construire pour former des coalitions et conflits à affronter.

Pour mieux comprendre la façon dont s'élaborent des alliances, des associations, voire même des coalitions, il faut donc s'intéresser au travail concret de persuasion que conduisent les acteurs. Plusieurs théories considèrent que les coalitions s'organisent en fonction d'intérêts (Richardson, 2013), d'idées (Sabatier et Weible, 2014), de paradigme (Haas, 1992), un peu comme si la coopération des acteurs allait de soi lorsqu'un socle commun existe et est partagé. Une telle hypothèse est contradictoire avec les travaux qui prennent au sérieux le dilemme de l'action collective et considèrent que l'action collective est toujours un problème à résoudre et non un phénomène naturel (Olson, 1987 ; Crozier et Friedberg, 1977). Aucune des propositions que nous avons étudiées empiriquement n'est portée dès son émergence par un large ensemble d'individus qui y adhèrent spontanément, naturellement et instantanément. Chaque proposition étudiée est d'abord portée par quelques acteurs dont le travail principal consiste justement à enrôler d'autres acteurs pour élargir le cercle des soutiens.

Le cas du tramway parisien est révélateur à plus d'un titre, dans la mesure où la proposition de tramway commence à se propager à partir de 1995, non parce que des acteurs y adhèrent spontanément grâce à des valeurs ou des croyances communes, mais grâce au travail concret de promotion que conduisent leurs porteurs. Ce travail est d'autant plus intéressant à observer qu'il s'opère dans un cadre concurrentiel où deux tracés sont en compétition. Il est d'ailleurs étonnant d'observer à quel point les coalitions qui vont se former autour de chaque tracé vont 
traverser toutes les organisations et ne recouvrent aucune division simple qui les rendrait compréhensibles autrement que par ce travail d'enrôlement. On trouve ainsi des défenseurs des deux tracés au sein de la Régie des transports parisiens, de la Ville de Paris, chez les élus mais aussi chez les techniciens, au sein de l'Agence d'urbanisme et même de I'Association des usagers des transports.

Dans ce monde concurrentiel des propositions, la policy analysis, c'està-dire le couplage d'une solution à un problème et aussi la production analytique et argumentaire qui l'accompagne, est un atout essentiel dans le travail de persuasion. Comme l'a montré un auteur comme Perelman (Perelman et Olbrechts-Tyteca, 2008), mais aussi comme Majone (1992), la construction de la vraisemblance d'une solution a d'abord à voir avec le travail d'argumentation qui la porte. Dans un monde sans évidence, la vraisemblance se gagne donc à coup d'arguments.

Ce travail argumentaire est d'autant plus important que les acteurs ne se soumettent pas qu'à un impératif de justification, ils doivent aussi pouvoir résister aux critiques qui sont faites à l'énoncé. Ce travail critique a lui aussi été particulièrement sous-estimé dans les travaux sur les politiques publiques. C'est tout l'intérêt des travaux de Boltanski (2009) d'avoir porté l'attention sur cette dimension critique. C'est une dimension que nous connaissons bien dans le monde de la recherche, qui veut qu'un article ou qu'une hypothèse doive passer l'épreuve de la critique pour être accepté. Mais un tel travail critique se retrouve chez les experts d'un secteur qui n'hésitent pas, lorsqu'une nouvelle proposition émerge, à souligner son absence de "fiabilité ", de "faisabilité ", de " cohérence ", etc. Plus largement, on retrouve ce travail critique dans les bureaucraties, où l'administration passe sous le joug de la critique toute nouvelle proposition. Et, bien évidement, l'espace politique est le lieu le plus significatif de la critique, du moins dans les démocraties: les critiques se structurent à la fois dans les controverses politiques, mais aussi dans le travail journalistique, etc.

$\mathrm{Au}$ fil de sa "carrière ", une proposition parcourt ainsi des mondes d'épreuves et tout énoncé qui n'aurait pas dressé des résistances suffisantes peut être amené à disparaître. Dans le travail que je conduis actuellement sur le gaz de schiste (Chateauraynaud et Zittoun, 2014), il 
apparaît clairement que la ministre de l'Environnement, qui avait initialement refusé un moratoire sur les permis d'explorer délivrés en France, refus appuyé sur un travail de justification, a modifié sa position lorsque son travail de justification a été mis à mal par les critiques.

Lorsque l'on s'intéresse aux pratiques concrètes des acteurs dans le monde de la fabrique des politiques publiques, on observe que, tout autant dans les espaces discrets que sous les projecteurs, les acteurs passent leur temps à argumenter, persuader, critiquer. La réussite de ces pratiques est indissociablement liée à la carrière des énoncés de propositions. Neustadt (1990), qui a examiné de très près l'activité quotidienne du président des États-Unis, en a conclu que le président passait son temps à chercher à persuader. Banfield (1961) avait lui aussi ouvert la voie en montrant l'importance du travail concret d'influence dans les organisations. L'approche pragmatique propose d'aller plus loin, pour faire de ces jeux d'acteurs associés aux jeux de langage les pratiques majeures qu'il faut observer pour comprendre ce qui fait qu'une proposition de politiques publiques émerge ou échoue.

La fabrique des énoncés de politiques publiques comme jeu de pouvoir

Les pratiques discursives ne sont pas seulement des pratiques qui entremêlent des jeux de langage et des jeux d'acteurs, elles sont également indissociables de jeux de pouvoir (Foucault, 1971). C'est un défaut classique des approches discursives ou langagières d'écarter les questions de pouvoir, comme si le pouvoir des arguments venait se substituer au pouvoir des acteurs. L'approche pragmatique des politiques publiques fait de la question du pouvoir une question centrale tout en considérant indissociable le pouvoir des acteurs de celui des arguments.

La question du pouvoir dans la fabrique des politiques publiques peut être abordée de nombreuses façons. Tout d'abord, il faut considérer qu'une proposition ne cherche pas seulement un problème à résoudre, elle cherche aussi un décideur à légitimer. En science politique, le débat a longtemps été de se demander s'il existait un décideur. D'une certaine façon, à chaque fois que les chercheurs ont voulu identifier un décideur, ils 
en ont conclu qu'il n'existait pas vraiment ou qu'ils étaient si nombreux à contribuer au processus décisionnel que le pouvoir devait être considéré comme diffus, éclaté, fragmenté et que personne n'avait vraiment décidé. Et pourtant, si les chercheurs ne peuvent identifier le "vrai » décideur, les acteurs n’hésitent pas à associer une proposition à un décideur.

Dans l'exemple du tramway parisien, il ne fait de doute pour personne que le maire est le décideur principal. Et pourtant, il existe de très nombreux arguments pour prouver que ce n'est pas tout à fait le cas, la responsabilité juridique par exemple incombant à l'État et à la Région. Cela n'empêche pas les acteurs de le penser. Bien plus, lorsque celui-ci a pris position sur le choix du tracé (il mettra plus de trois ans à se décider entre deux tracés), tous les acteurs qui l'entourent, y compris ceux censés avoir du pouvoir, reconnaissent sa prise de position comme faisant office de décision. En réalité, il nous faut non pas nous enfermer dans la quête d'un "vrai » décideur, mais comprendre toute l'importance que revêt l'opération de désignation d'un décideur. Le tramway ne voit le jour que lorsque le maire s'approprie le projet et en devient effectivement le propriétaire.

Gusfield (2008) avait souligné que le travail de définition d'un problème s'organisait d'abord autour de la désignation d'un responsable. Kingdon (2010) avait lui aussi montré qu'une situation ne devient un problème public que lorsqu'elle est définie comme devant être résolue par une autorité publique. De la même façon, on peut dire qu'un énoncé de solution ne prend forme qu'à partir du moment où un "décideur " se l'attribue. Ce travail est essentiel car la reconnaissance de la figure du décideur est aussi une forme de reconnaissance de son pouvoir et de sa position dans l'espace sociopolitique. Évidemment, cette position ne lui est pas acquise, il doit la gagner car, si le " décideur " échoue, il se met en danger.

Mais le "décideur " n'est pas le seul à décider, une proposition est indissociable de ses "propriétaires ", c'est-à-dire de tous ceux qui se sont appropriés la mesure et qui en revendiquent l'autorité. Le débat autour du choix du tracé du tramway est d'autant plus intéressant qu'il traverse, comme on l'a vu, toutes les organisations. Dans chaque organisation, l'appropriation de l'un ou l'autre des tracés est aussi un moyen pour 
mettre à l'épreuve des positions de pouvoir, le vainqueur se voyant renforcé dans sa position. De ce point de vue, les acteurs lient une petite part de leur pouvoir et de leur identité à ce tracé. S'ils s'imposent, ils pourront en revendiquer le succès et le pouvoir qu'ils en tirent. La réussite de leur tracé n'est pas seulement le révélateur de leur pouvoir, elle contribue à le façonner. Ainsi la RATP voit s'opposer sur le tracé sa direction des Bus à sa direction des Métros, le tramway étant un être hybride. Si cette dernière est considérée comme plus puissante, c'est en fait la première qui va l'emporter, ce qui lui a permis de se repositionner dans les jeux de pouvoir habituels au sein de l'organisation.

Une façon de se représenter cette question des pouvoirs est de considérer, d'une part, qu'il existe une répartition des "territoires " de politiques publiques entre différents propriétaires et, d'autre part, que chaque proposition est l'occasion de mettre à l'épreuve ce "cadastre", c'est-à-dire cette cartographie où sont identifiées les frontières en fonction de leur propriétaire. Ainsi, la division du travail que génère tout processus de bureaucratisation se construit autour d'une appropriation d'un secteur spécifique. Le ministère du Logement est le propriétaire de toutes les politiques publiques qui ont à voir avec la question du logement. Le ministère de la Recherche avec ce qui concerne la recherche, etc. Chaque ministère ne s'organise pas seulement à partir de la division en secteurs, il structure une sorte de cadastre avec une définition (toujours friable et poreuse) des frontières, dans lequel il assure sa domination et la répartition des positions et des pouvoirs en son sein. Dans nos travaux, nous avons pu ainsi observer les conflits entre le ministère du Logement et celui des Affaires sociales pour savoir si la proposition de "logement d'urgence " n'était pas un " hébergement " déguisé. Au-delà de la simple lutte sémantique qui distingue les deux concepts, $c^{\prime}$ est d'abord un enjeu de propriétaire car, si le "logement d'urgence" est requalifié en hébergement, il tombe automatiquement dans l'escarcelle du ministère des Affaires sociales.

Chaque nouvelle solution est porteuse elle-même d'une configuration spécifique des pouvoirs, et son intégration à une politique publique existante doit être comprise comme un problème dans la mesure où elle vient modifier la répartition des pouvoirs en présence. C'est aussi parce qu'elle provoque une modification des asymétries, que les acteurs 
s'engagent avec force dans les conflits pour ou contre une nouvelle proposition. Il est donc essentiel de comprendre à quel point une politique publique est toujours un enjeu de pouvoir et que son changement est porteur d'une possibilité de redistribution de ressources qui peut porter à conséquence.

Conclusion : L'approche pragmatique pour saisir la dimension politique des politiques publiques

L'approche pragmatique des politiques publiques est donc d'abord une approche qui se propose de saisir les processus de fabrique et de transformation des politiques publiques à travers la description micro des pratiques concrètes des acteurs, et en s'intéressant tout particulièrement à la façon dont les acteurs associent à leur proposition, une solution à résoudre, des acteurs à agréger, des pouvoirs à répartir et des arguments pour rationaliser.

La sociologie pragmatique s'est beaucoup focalisée sur la dynamique des "causes" et des "problèmes". Elle a permis de mieux comprendre l'importance du travail de définition dans le processus de mise à l'agenda. Elle a permis également de s'attacher à une activité politique majeure, celle de la contestation du pouvoir et de la mise en désordre. Boltanski (2008) montre comment le travail de définition des problèmes participe à " rendre inacceptable la société ", c'est-à-dire à donner à voir l'injustice et les problèmes d'une société. On peut dire, pour prendre un vocabulaire de la science politique, que la définition des problèmes publics contribue à dessiner l'image d'une société en désordre, d'une société au futur problématique et qui, si rien n'est fait, peut devenir apocalyptique et, par là-même, qu'elle contribue à produire du désordre en contestant l'ordre établi, celui d'un gouvernement qui n'agit pas.

L'approche pragmatique des politiques publiques s'intéresse davantage à l'activité du gouvernement et voit dans l'observation concrète des pratiques de ce dernier le moyen de mieux comprendre les conditions d'émergence, de succès, mais aussi d'échec des propositions de politiques publiques. Plus encore, elle permet de comprendre le rôle majeur de la définition des solutions comme la façon dont les individus tentent de 
dessiner une société future qui retrouve de l'ordre, c'est-à-dire une société où les problèmes sont résolus, où le futur est réenchanté, où les décideurs se remettent à décider, etc. Le dessin d'une société future réordonnée n'est pas seulement la production d'une fiction, il contribue en lui-même à produire de l'ordre politique.

En cela, l'approche pragmatique permet de comprendre que l'analyse des politiques publiques n'est ni un art, ni une science, mais une activité politique d'apprivoisement des problèmes et du désordre et de production d'un ordre toujours instable et éphémère.

\section{Bibliographie}

ANDERSON J.E., 1979, Public Policy-Making, Holt, Rinehart and Winston.

BANFIELD E.C., 1961, Political influence, Transaction Publishers.

BARDACH E. et PATASHNIK E.M., 2015, A Practical Guide for Policy Analysis: The Eightfold Path to More Effective Problem Solving, $5^{\text {th }}$ Revised edition, SAGE Publications Inc.

BARTHE Y., DE BLIC D., HEURTIN J.-P., LAGNEAU E., LEMIEUX C., LINHARDT D., MOREAU DE BELLAING C., REMY C., TROM D., 2014, "Sociologie pragmatique : mode d'emploi », Politix, 3 : 175-204.

BOLTANSKI L., 2008, Rendre la réalité inacceptable, Demopolis.

BOLTANSKI L., 2009, De la critique: Précis de sociologie de l'émancipation, Gallimard.

BOUSSAGUET L., JACQUOT S. et RAVINET P. (éds), 2015, Une " French touch » dans I'analyse des politiques publiques ?, Paris, Presses de Sciences Po.

COMMAILLE J., 2004, "Sociologie de l'action publique ", in BOUSSAGUET L., JACQUOT S. et RAVINET P., Dictionnaire des politiques publiques, Paris, Presses de Sciences Po : 413-420.

CHATEAURAYNAUD F. et ZITTOUN P., 2014, " The future they want-or do not want: Shale gas opponents vs. proponents between local motives and global scenarios ", in $9^{\text {th }}$ International Conference on Interpretive Policy Analysis, Wageningen, The Netherlands. 
COHEN M.D., MARCH J.G. et OLSEN J.P., 1972, "A garbage can model of organizational choice ", Administrative science quarterly : 1-25.

CROZIER M. et FRIEDBERG E., 1977, L'acteur et le système, Coll. Points, Paris, Seuil.

DAHL R.A. et LINDBLOM C.E., 1963, Politics, Economics, and Welfare: Planning and Politico-economic Systems Resolved Into Basic Social Processs, Harper \& Row, Publ.

DEWEY J., 2010, Le public et ses problèmes, Éditions Gallimard.

DYE T.R., 1972, Understanding public policy, Englewood Cliffs, N.J., Prentice-Hall.

FISCHER F. et GOTTWEIS H., 2012, The Argumentative Turn Revisited: Public Policy As Communicative Practice, Duke University Press.

FOUCAULT M., 1971, L'ordre du discours, Paris, Gallimard.

FOUCAULT M., 1975, Surveiller et punir : naissance de la prison, Bibliothèque des histoires, Paris, Gallimard.

GUSFIELD J., 2008, La culture des problèmes publics: L'alcool au volant: la production d'un ordre symbolique, Paris, Économica.

HALPERN C., HASSENTEUFEL P. et ZITTOUN P., à paraître, Policy Analysis in France, Policy Press.

HAAS P.M., 1992, « Introduction: epistemic communities and international policy coordination », International organization, 46(1) : 1-35.

HEAD B.W., 2008, "Wicked problems in public policy ", Public Policy, 3(2) : 101118.

HECLO H., 1972, " Policy Analysis », British Journal of Political Science, 2(1) : 83-108.

HOWLETT M., 2009, " Governance modes, policy regimes and operational plans: A multi-level nested model of policy instrument choice and policy design ", Policy Sciences, 42(1) : 73-89.

HUDSON J. et LOWE S., 2014, Understanding the policy process, $2^{\mathrm{e}}$ éd., The Policy Press.

JAMES W., 1995, Pragmatism, New name for some Old Ways of Thinking, Mineola, Courier Dover Publication.

JOBERT B. et MULLER P., 1987, L'État en action: politiques publiques et corporatismes, Presses universitaires de France. 
JONES C.O., 1984, An Introduction to the Study of Public Policy, $3^{\mathrm{e}}$ éd. révisée, Wadsworth Publishing Co Inc.

KINGDON J.W., 2010, Agendas, Alternatives, and Public Policies, Update Edition, with an Epilogue on Health Care, $2^{\mathrm{e}}$ éd., Longman.

LASSWELL H.D., 1951, The Policy Sciences: Recent Developments in Scope and Method, Stanford University Press.

LASSWELL H.D., 1971, Preview of Policy Sciences, Elsevier Science Ltd.

LASCOUMES P. et LE GALES P., 2007, Sociologie de l'action publique, Coll. 128, Paris, Armand Colin.

LATOUR B., 1992, Aramis ou l'amour des techniques, Paris, La Découverte.

LINDBLOM C.E., 1959, "The Science of "Muddling Through" ", Public Administration Review, 19(2) : 79-88.

LINDBLOM C.E., 1979, "Still muddling, not yet through ", Public administration review, 39(6) : 517-526.

LINDBLOM C.E. et WOODHOUSE E.J., 1992, The Policy Making Process, 3 éd., Pearson.

MAJONE G., 1992, Evidence, Argument and Persuasion in the Policy Process, nouvelle éd., Yale University Press.

NEUSTADT R.E., 1990, Presidential Power and the Modern Presidents: The Politics of Leadership from Roosevelt to Reagan, The Free Press.

NEVEU E., 2015, Sociologie politique des problèmes publics, Paris, Armand Colin.

OLSON M., 1987, Logique de l'action collective, Paris, PUF.

PERELMAN C. et OLBRECHTS-TYTECA L., 2008, Traité de l'argumentation: La nouvelle rhétorique, $6^{\mathrm{e}}$ éd., université de Bruxelles.

PETERS G.B., 2015, Advanced Introduction to Public Policy, Edward Elgar Publishing Ltd.

RICHARDS D. et SMITH M.J., 2002, Governance and public policy in the United Kingdom, Oxford University Press.

RICHARDSON J., 2013, Policy Styles in Western Europe (Routledge Revivals), Routledge.

SABATIER P.A. et Weible C.M., 2014, Theories of the Policy Process, 3 éd., Westview Press. 
SABATIER P.A., 1998, Theories of the Policy Process, Lieu, Westview Press Inc.

SIMON H.A., 1997, Administrative Behavior, 4th Edition, $4^{e}$ éd. révisée, $\mathrm{S} \& \mathrm{~S}$ International.

SPECTOR M. et KITSUSE J.I., 1973, "Social problems: A re-formulation », Social problems, 21(2) : 145-159.

STONE D., 2001, Policy Paradox: The Art of Political Decision Making, 2 éd. révisée, WW Norton \& Co.

WILDAVSKY A., 1964, The politics of the budgetary process, Boston, Little, Brown.

WILDAVSKY A., 1969, "Rescuing policy analysis from PPBS ", Public Administration Review, 29(2) : 189-202.

WILDAVSKY A., 1987, Speaking Truth to Power: The Art and Craft of Policy Analysis, Reprint, New Brunwick (NJ), Transaction Publishers.

WILDAVSKY A., 2006, Budgeting And Governing, New edition, Transaction Publishers.

WITTGENSTEIN L., 2014, Recherches philosophiques, Paris, Gallimard.

ZITTOUN P., 2009, « Understanding policy change as a discursive problem », Journal of Comparative Policy Analysis, 11(1) : 65-82.

ZITTOUN P., 2013, "Entre définition et propagation des énoncés de solution ", Revue française de science politique, 63(3) : 625-646.

ZITTOUN P. et DEMONGEOT B., 2010, « Debates in French policy studies: from cognitive to discursive approaches », Critical policy studies, 3(3-4) : 391406.

ZITTOUN P. et PETERS B.G., 2016, Contemporary Approaches to Public Policy: Theories, Controversies and Perspectives, Palgrave Macmillan.

\title{
NANANON
}

\author{
Philippe Zittoun est chercheur en science politique \\ LAET-ENTPE, université de Lyon \\ E-mail : pzittoun@gmail.com
}

